\title{
Evaluation of valid reference genes during stimulation with static magnetic fields in human myoblast cultures
}

\author{
JENS STERN-STRAETER ${ }^{1 *}$, GABRIEL ALEJANDRO BONATERRA ${ }^{2 *}$, STEFANIE ZÜGEL ${ }^{2}$, \\ STEFAN S. KASSNER ${ }^{1}$, KARL HÖRMANN ${ }^{1}$, RALF KINSCHERF ${ }^{2 * *}$ and ULRICH REINHART GOESSLER ${ }^{1 * *}$ \\ ${ }^{1}$ Department of Otolaryngology, Head and Neck Surgery, University Hospital Mannheim, University of Heidelberg, \\ 68167 Mannheim; ${ }^{2}$ Institute for Anatomy and Cell Biology, Department of Medical Cell Biology, 35032 Marburg, Germany
}

Received October 5, 2009; Accepted November 23, 2009

DOI: $10.3892 / \mathrm{mmr} 000000246$

\begin{abstract}
Investigation of gene expression using real-time PCR (qRT-PCR) requires normalization with genes that are continuously expressed (reference genes; RGs). For accurate measurements, it is exceedingly important that RG expression is invariant under the investigated experimental conditions. It has recently become evident that RG expression may vary considerably under different culture conditions, which results in inaccurate qRT-PCR measurements. Static magnetic fields (SMFs) have been shown to enhance myogenic cell differentiation in the rat cell line L6, and may also induce differentiation in human myoblast cultures. In order to perform precise qRT-PCR measurements in human myoblast cell cultures stimulated with SMFs, one prerequisite is to find the most suitable RG. In this study, qRT-PCR was used to investigate the gene expression of six widely used RGs in human myoblast cell cultures stimulated with SMFs, with the aim of identifying the most stable among them. The mRNA concentration of $\beta$-actin (ACTB), $\beta$-2-microglobulin (B2M), glyceraldehyde-3-phosphate dehydrogenase (GAPDH), peptidylprolyl isomerase A (PPIA), TATA box binding protein (TBP) and ribosomal protein, large, P0 (RPLPO) were quantified, and the most suitable RGs were identified using the geNorm and NormFinder software programs. Results were verified by BestKeeper software. mRNA expression of the following genes of interest was analyzed: myosin, heavy chain 1, skeletal muscle, adult (MYH1); myosin, heavy chain 3, skeletal muscle, embryonic (MYH3); myosin, heavy chain 8, skeletal muscle, perinatal (MYH8), as well as the immunoreactivity of MYH1
\end{abstract}

Correspondence to: Dr Jens Stern-Straeter, Department of Otolaryngology, Head and Neck Surgery, University Hospital Mannheim, D-68135 Mannheim, Germany

E-mail: jens.stern-straeter@umm.de

*Contributed equally

${ }^{* *}$ Senior authors

Key words: real-time polymerase chain reaction, reference gene, skeletal muscle, satellite cells, static magnetic fields
(irMYH1). Using geNorm, PPIA and B2M were found to be the most stable genes, followed by GAPDH. NormFinder identified PPIA as the most stable gene, followed by B2M and GAPDH. Finally, BestKeeper revealed TBP and PPIA to be the most stable genes, while B2M was ranked third.

\section{Introduction}

The real-time polymerase chain reaction (qRT-PCR) technique has become a routine technique for gene expression analysis. It allows accurate high-output RNA expression analysis in molecular biology thanks to its increased sensitivity and specificity over the classical semi-quantitative RT-PCR technique $(1,2)$. However, certain methodological disadvantages exist; for example, data normalization remains problematic and is subject to frequent criticism. Normalization is essential to control experimental errors between samples that can be introduced at a number of stages throughout the procedure. Reference genes (RGs), commonly called housekeeping genes (HKGs), are frequently chosen from the literature and applied across a variety of experimental conditions. However, some of these conditions may induce differences in the HKG's own expression (3). Therefore, it is essential to validate potential RGs in order to establish whether they are appropriate for a specific experimental purpose (4).

Unexpected changes in RG expression may occur during various stages of an experiment and need to be corrected between samples (4-6). Therefore, proper selection of the most stable RG is essential. In order to select the best RG for an investigation, one must consider the following aspects: the abundance and amplification efficiency of the RG should be similar to that of the genes of interest, and the expression of the $\mathrm{RG}$ must remain constant across the investigated experimental conditions (7). Changes in RG expression under the assessed experimental conditions may result in methodological errors and incorrect conclusions about the analyzed biological effects. Therefore, verification of the most suitable RG under the investigated conditions (stimulated and control groups) is an important step in qRT-PCR experiments (8).

The aim of skeletal muscle tissue engineering is the regeneration of skeletal muscle loss by the expansion and differentiation of stem cells that are able to differentiate into the myogenic cell line in vitro. Satellite cells, also called 
myoblasts, fulfill the requirements for this approach, since they are easily obtainable through muscle biopsies and can differentiate into skeletal muscle tissue (9). However, one must also develop techniques which induce the stable differentiation of these cells in the direction of functional myofibers in expanded cell cultures. These stimuli have to be practical options for a clinical setting, and must therefore avoid the use of possible mutagenic substances (e.g., Matrigel ${ }^{\circledR}$ ) and genetic manipulation (10). Coletti et al showed that static magnetic fields (SMFs) with an intensity of $\pm 80 \mathrm{mT}$ promote myogenic cell differentiation in the immortal rat cell line L6 in terms of increased accumulation of actin and myosin and the formation of large multinucleate myotubes (11). Based on these observations, it is of interest to analyze the effect of SMFs in human myoblast cell cultures in order to identify a possible clinically suitable differentiation stimulus. In order to perform exact qRT-PCR experiments in this environment, one must identify the suitable RG. Therefore, we evaluated six commonly used RGs during stimulation experiments with SMFs in human myoblast cell cultures.

\section{Materials and methods}

Cell culture. Skeletal muscle biopsies were obtained during head and neck surgery from 15 patients with a median age of 58 years. The study protocol was approved by the Ethics Committee of the Medical Faculty Mannheim of the University of Heidelberg, Germany, and all patients gave their informed written consent. Satellite cells, also called myoblasts, were dissociated from the minced muscles by digestion with collagenase B (Roche, Mannheim, Germany) for $60 \mathrm{~min}$ and $0.05 \%$ trypsin- $0.02 \%$ EDTA (PromoCell, Heidelberg, Germany) for $45 \mathrm{~min}$ at $37^{\circ} \mathrm{C}$, then filtered through a sterile $70-\mu \mathrm{m}$ cell strainer (Becton Dickinson, Franklin Lakes, NJ, USA) and purified with the pre-plating technique recently described (12). Purity of myoblast cultures $(>80 \%)$ was evaluated by anti-desmin immunostainings. Cells were grown on $0.2 \%$ gelatine-coated culture flasks (Sigma, Deisenhofen, Germany) in Ham's F10 growth medium containing $1 \%$ penicillin/streptomycin/ fungizone-solution (PSF), $2 \mathrm{mM} \mathrm{L-glutamine} \mathrm{(PromoCell)}$ and $10 \%$ foetal bovine serum (FBS) (PAA Laboratories, Linz, Austria). This medium was referred to as 'growth medium' (GM). The GM was changed every $72 \mathrm{~h}$, and cells were cultured at $37^{\circ} \mathrm{C}$ in a humidified atmosphere of $5 \% \mathrm{CO}_{2}$ and $95 \%$ air.

Differentiation of skeletal muscle myoblasts. Satellite cells were grown to $60 \%$ confluence, washed with phosphatebuffered saline (PBS) and induced to differentiate by a change in differentiation medium (DM) consisting of minimal essential medium (MEM) (PromoCell) supplemented with $2 \%$ horse serum (PAA Laboratories), $2 \mathrm{mM}$ L-glutamine and PSF (PromoCell).

Immunohistochemistry. Immunohistochemical characterization was carried out on cells grown on chamber culture slides (BD Falcon, Franklin Lakes, NJ, USA). Myoblasts were identified using antibodies to myosin heavy chain 1 (MYH1) (Abcam, Cambridge, UK) as a differentiation marker. The MYH1-antibody was used in a solution of $1 / 20$, followed by a specific biotinylated secondary antibody.
The peroxidase reaction was performed using aminoethylcarbazol (Dako) as a chromogen. Endogenous peroxidase was blocked with $0.3 \%$ hydrogen peroxide for $30 \mathrm{~min}$. Sections were washed with PBS and incubated with normal sheep serum in PBS for $30 \mathrm{~min}$ at room temperature to block nonspecific antibody reaction. Nuclei were counterstained with Harris' haematoxylin. Light microscopy investigation was performed using a Zeiss Axiophot microscope.

Fusion index determination. To analyze differentiation, the number of nuclei in MYH1-positive cells was counted and expressed as a percentage of the total number of nuclei analyzed. The fusion index irMYH1 was determined at day 8 of cultivation by dividing the number of nuclei within myotubes ( $\geq 2$ nuclei) by the total number of nuclei analyzed in MYH1-positive myotubes.

Static magnetic field exposure. SMFs were produced by $4 \times 4 \mathrm{~cm}$ neodymium magnetic plaques with a magnetic field of $80 \pm 5 \mathrm{mT}$, verified by a gaussmeter. The magnetic plaques were placed underneath the culture dishes at a 1-mm distance from the cell monolayer culture. For details see Coletti et al (11). The magnetic fields were axial with the magnetic North vector crossing the cells (the pole of the magnet which is repulsed by the Earth's North magnetic pole). Control samples were grown simultaneously and induced to differentiate under identical experimental conditions without the presence of SMFs. Magnets did not produce temperature variation.

RNA isolation. Total RNA was isolated using the RNeasy Mini Kit (Qiagen GmbH, Hilden, Germany) according to the manufacturer's instructions. RNA concentration, purity and integrity were determined by A260 and A280 (A260/A280=1.7-2.0) measurements using a NanoDrop 8000 Spectrophotometer (Thermo Scientific, Schwerte, Germany) and Agilent 2100 Bioanalyzer (Agilent Technologies, Waldbronn, Germany).

cDNA synthesis and real-time PCR. An aliquot of $0.5 \mu \mathrm{g}$ total RNA was treated with 1 unit DNase (Fermentas, St. Leon-Rot, Germany) for $30 \mathrm{~min}$ at $37^{\circ} \mathrm{C}$. Reverse transcription of RNA $(0.5 \mu \mathrm{g})$ was performed with an oligo (dT)12-18 primer, 200 units of Superscript II (Invitrogen, Karlsruhe, Germany) and 24 units of Ribo Lock ${ }^{\mathrm{TM}}$ RNase inhibitor (Fermentas) for $1 \mathrm{~h}$ at $42^{\circ} \mathrm{C}$. cDNA was used for quantitative RT-PCR (qRT-PCR) analysis. All cDNA probes were analyzed for skeletal muscle $\alpha 1$ actin (ACTA1), NM_001100; myosin, heavy chain 1, skeletal muscle, adult (MYH1), NM_005963; myosin, heavy chain 3, skeletal muscle, embryonic (MYH3), NM_002470; myosin, heavy chain 8, skeletal muscle, perinatal (MYH8), NM_002472; and the RGs $\beta$-actin (ACTB), NM_001101; $\beta$-2-microglobulin (B2M), NM_004048; glyceraldehyde-3-phosphate dehydrogenase (GAPDH), NM_002046; peptidylprolyl isomerase A (cyclophilin A) (PPIA), NM_203430; large ribosomal protein P0 (RPLPO), NM_001002; and TATA box binding protein (TBP), NM_003194. The QuantiTect/PrimerAssays were purchased from Qiagen GmbH. cDNA was amplified with Brilliant ${ }^{\circledR}$ II SYBR ${ }^{\circledR}$ Green QRT-PCR Master Mix (Stratagene, Waldbronn, Germany). The thermal profile consisted of 1 cycle at $50^{\circ} \mathrm{C}$ for $2 \mathrm{~min}$ followed by 1 cycle at $95^{\circ} \mathrm{C}$ for $2 \mathrm{~min}$ and 45 cycles at $95^{\circ} \mathrm{C}$ for $15 \mathrm{sec}$ and at $60^{\circ} \mathrm{C}$ for $1 \mathrm{~min}$. 
Amplification was performed using the Mx3005P ${ }^{\text {TM }}$ QPCR System (Stratagene-Agilent Technologies). For relative quantification, a standard curve was generated in every individual run. Total RNA was pooled from muscle biopsies of healthy human volunteers. Reverse transcription was performed, and a serial dilution of cDNA was used to perform the calibration curve. Data were analyzed using the relative standard curve method. For each unknown sample, the relative amount was calculated using linear regression analysis from their respective standard curves, and data were analyzed using Mx3005P analysis software (Stratagene-Agilent Technologies). The efficiencies of all genes of interest and those of the RGs were calculated in every individual run.

Analysis of expression stability. The qRT-PCR data were analyzed using the Mx3005P ${ }^{\mathrm{TM}}$ QPCR System. For stability comparisons of candidate RGs within sample groups, the software geNorm version 3.4 (13) (Visual Basic application tool for Microsoft Excel), NormFinder (14) and BestKeeper (15) were used according to the developer's recommendations.

GeNorm uses a gene-stability measure M, which is defined as the average pair-wise variation between a particular gene and all other control genes. It calculates the optimal number of genes necessary for normalization of a target gene and combines them into a normalization factor (NF). NormFinder uses a model-based approach for the estimation of expression, and enables the identification of the single best genes, as well as providing the order of ranking. BestKeeper determines the best suited RGs and combines them into an index (BK). This index can be compared with further target genes to decide whether they are differentially expressed under certain conditions. NormFinder has an additional option to define groups, which was applied to compare the effect of the treatment of myoblasts with SMFs.

Statistical analysis. SigmaPlot software was used to carry out statistical analysis using the paired Student's t-test or the Mann-Whitney U test, as well as Pearson's product moment correlation tests of gene expression among experimental groups. Gene expression results were expressed as the mean. A P-value $<0.05$ was considered statistically significant.

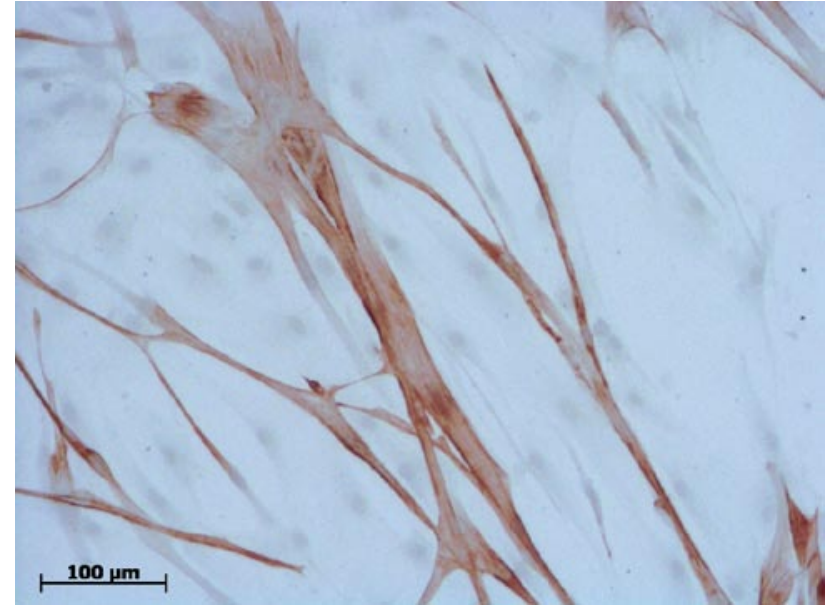

Figure 1. Immunohistochemical staining of human myoblast cultures with anti-myosin heavy chain 1.

\section{Results}

RNA quality. The concentration, purity and integrity of the RNA samples were investigated. RNA purity was measured using the NanoDrop ${ }^{\circledR}$ Spectrophotometer (NanoDrop Technologies). Based on the absorbance ratio at $260 \mathrm{~nm} / 280 \mathrm{~nm}$ (mean $\pm \mathrm{SD}$, $1.90 \pm 0.05)$, all RNA samples were pure and protein-free. The mean ( \pm SEM) A260/230 ratio of $1.90 \pm 0.20$ (range 1.65-2.10) indicated that the RNA was free of phenol and ethanol. RNA integrity was assessed by the calculation of RIN values using the Agilent 2100 Bioanalyzer (myoblast cultures treated with and without SMFs revealed RIN values between 6.0 and 8.0).

Immunohistochemistry. In order to confirm that the primary cells utilized were of muscle origin and did not change phenotype during cultivation, cell cultures were examined by immunohistochemistry using specific monoclonal antibodies against desmin and MYH1 (Fig. 1). A fusion index was calculated, and after 8 days MYH1 was detected in myotubes in cell cultures treated with SMFs, DM and GM. SMF treatment was significantly effective in myoblasts cultured with GM or DM (Fig. 2).

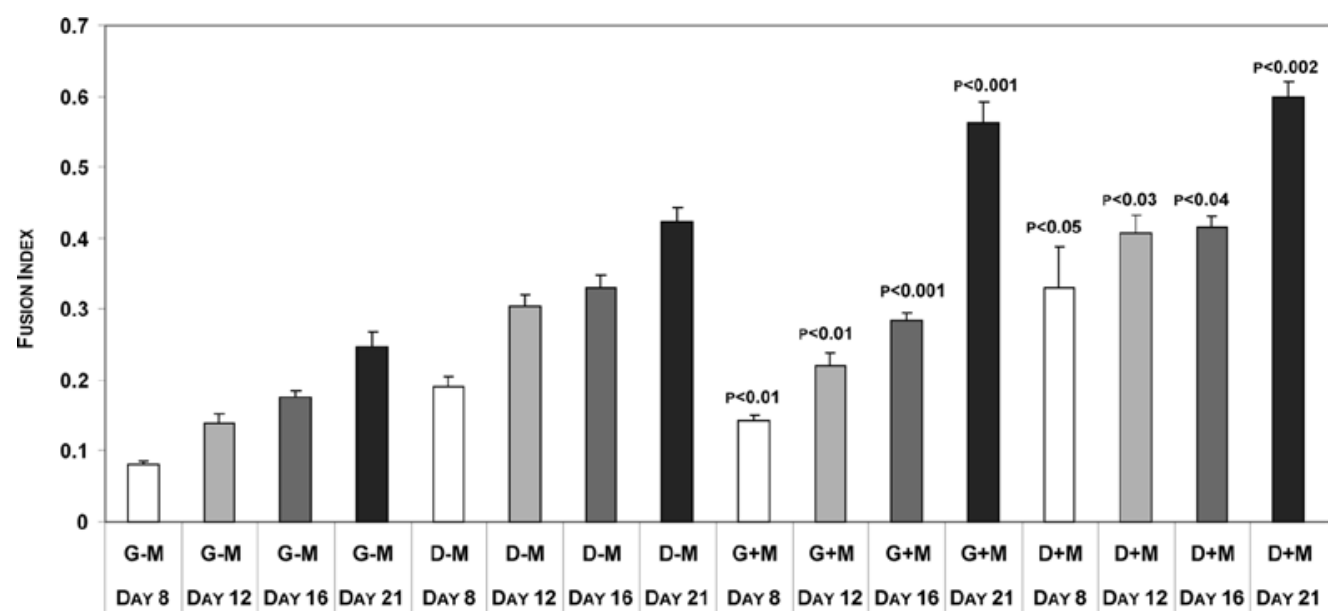

Figure 2. Quantification of myotube fusion. The results are expressed as a ratio of the number of nuclei in the myotubes to the number of total nuclei. Fusion values are the mean \pm SEM, - M vs. + M, of three experiments. D, differentiation media; G, growth media; +M, stimulated with SMFs, $-\mathrm{M}$, not stimulated with SMFs. 


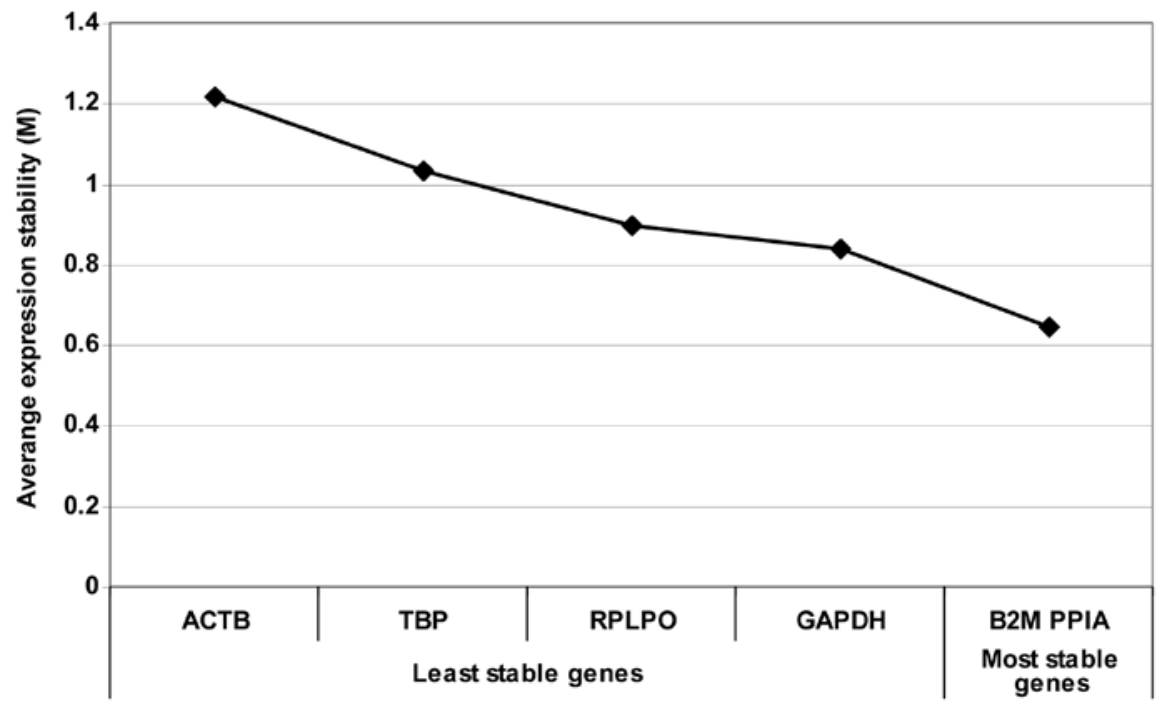

Figure 3. Average expression stability values of reference candidate genes. The gene expression stability of six candidate reference genes (RGs) was determined using geNorm analysis in order to enable the selection of the most suitable RGs for normalization in SMF-treated myoblast cultures. Results are presented according to the output file of the program (13). The least stable genes were identified by calculating the average expression stability measure M. The $\mathrm{x}$-axis from left to right indicates the ranking of the genes according to their M-value.

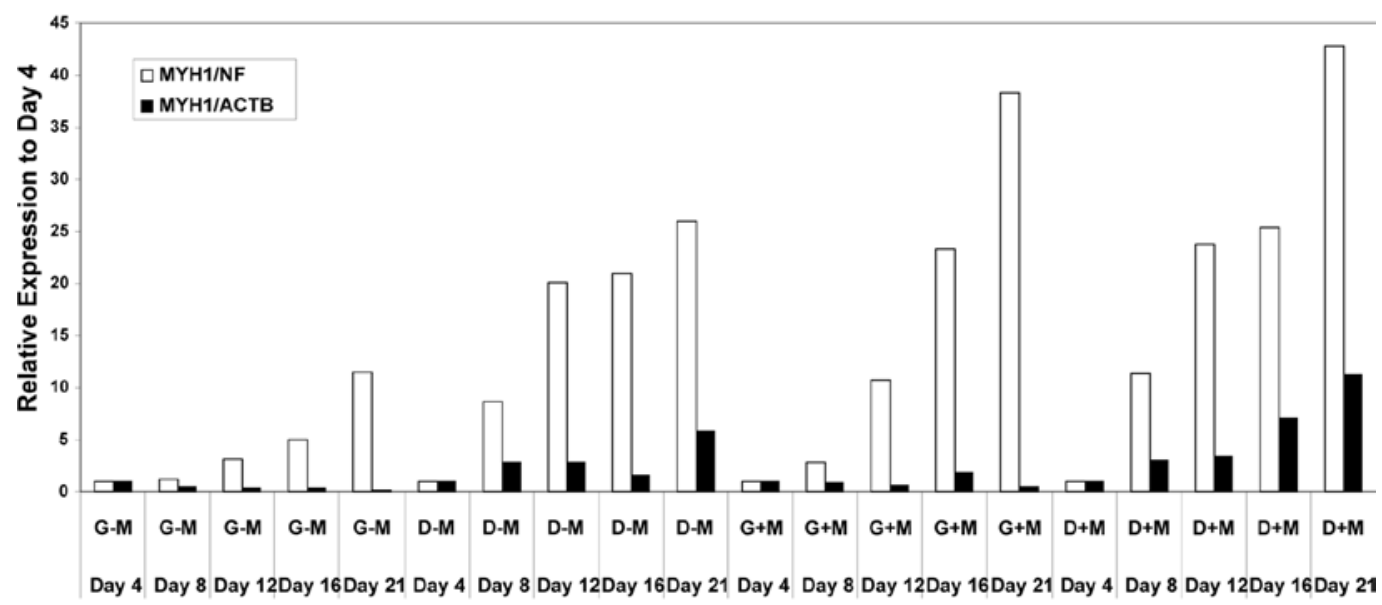

Figure 4. Comparison of the expression of the genes of interest normalized with the normalization factor (NF) and the worst RG candidate (ACTB). Results are the relative expression of MYH1, calculated 4 days after exposure to different periods of SMF treatment.

Stability of RGs within sample groups. Stability of the six RGs was first assessed for each sample group. Gene expression levels were measured by qRT-PCR, and the expression stabilities were evaluated by the three most commonly used software-based methods: geNorm (13), NormFinder (14) and BestKeeper (15).

geNorm. The average expression stability (M) was calculated by the geNorm software and is shown in Fig. 3. The M-value specifies the constancy of the gene expression, with a low M-value indicating high expression stability. According to the M-value, a ranking of the RGs was calculated and gene stability expression was identified. Based on the M-value, all the RGs reached the geNorm arbitrary cut-off level of 0.5 for stability (Fig. 3). This implies that the use of any of these RGs for normalization is appropriate. Successive elimination of the less stable genes generated a ranking of genes according to their M-values and resulted in the identification of PPIA and $\mathrm{B} 2 \mathrm{M}$ as the two most stable genes. In addition to the
Table I. Ranking order of the six candidate RGs using the NormFinder program to calculate their expression stability.

\begin{tabular}{lcc}
\hline Gene name & Stability value & Standard error \\
\hline PPIA & 0.267 & 0.087 \\
B2M & 0.379 & 0.087 \\
GAPDH & 0.395 & 0.087 \\
RPLPO & 0.644 & 0.108 \\
TBP & 0.739 & 0.118 \\
ACTB & 0.995 & 0.148
\end{tabular}

average gene expression stability $\mathrm{M}$, geNorm calculates a normalization factor (NF) based in all the RGs, taking into account the variable as the pair-wise variation between two sequential normalization factors (data not shown). 
Table II. Descriptive statistics of six RGs based on their crossing point (CP) values.

\begin{tabular}{|c|c|c|c|c|c|c|c|c|}
\hline & \multirow[b]{2}{*}{ PPIA } & \multirow[b]{2}{*}{ B2M } & \multirow[b]{2}{*}{ TBP } & \multirow[b]{2}{*}{ RPLPO } & \multirow[b]{2}{*}{ GAPDH } & \multirow[b]{2}{*}{ АCTB } & \multicolumn{2}{|c|}{ BestKeeper } \\
\hline & & & & & & & $n=6$ & $n=2$ \\
\hline $\mathrm{n}$ & 21 & 21 & 21 & 21 & 21 & 21 & 21 & 21 \\
\hline GeoM (CP) & 24.41 & 16.69 & 23.16 & 16.30 & 16.08 & 15.88 & 18.44 & 19.81 \\
\hline ArithM (CP) & 24.41 & 16.70 & 23.16 & 16.31 & 16.08 & 15.89 & 18.44 & 19.81 \\
\hline $\operatorname{Min}(\mathrm{CP})$ & 23.77 & 15.85 & 22.51 & 15.02 & 15.43 & 15.21 & 17.75 & 19.25 \\
\hline $\operatorname{Max}(\mathrm{CP})$ & 25.18 & 17.68 & 24.45 & 17.44 & 17.20 & 16.76 & 19.48 & 20.81 \\
\hline $\mathrm{SD}( \pm \mathrm{CP})$ & 0.31 & 0.31 & 0.45 & 0.33 & 0.33 & 0.36 & 0.32 & 0.30 \\
\hline $\mathrm{CV}(\% \mathrm{CP})$ & 1.27 & 1.89 & 1.94 & 2.00 & 2.06 & 2.26 & 1.71 & 1.53 \\
\hline
\end{tabular}

In the two last columns, the BestKeeper (BK) index is computed together with the same descriptive parameters, either for six genes (PPIA, B2M, TBP, RPLPO, GAPDH and ACTB) or for two genes after removal of TBP, RPLPO and GAPDH. n, number of samples; GeoM (CP), the geometric mean of CP; ArithM (CP), the arithmetic mean of CP; Min (CP) and Max (CP), the extreme values of CP; Min (x-fold) and Max ( $\mathrm{x}$-fold), the extreme values of expression levels expressed as an absolute $\mathrm{x}$-fold over- or under-regulation coefficient; $\mathrm{SD}( \pm \mathrm{CP})$, the standard deviation of the $\mathrm{CP} ; \mathrm{CV}(\% \mathrm{CP})$, the coefficient of variance expressed as a percentage of the CP level; SD ( $\pm \mathrm{x}$-fold), standard deviation of the absolute regulation coefficients. Results from three independent experiments are shown.

Table III. Pearson's correlation between the state of myoblast differentiation (fusion index from irMYHC1) and the expression of ACTA1, MYOG or MYH3, normalized with NF, calculated with RPLPO and TBP by GeNorm and RGs normalized with the same NF.

\begin{tabular}{|c|c|c|c|c|c|c|c|c|c|c|c|c|}
\hline & & ACTA1 & MYH1 & MYH3 & MYH8 & PPIA & B2M & TBP & RPLPO & GAPDH & ACTB & $\mathrm{NF}$ \\
\hline \multirow[t]{3}{*}{ IC-MYH1 } & $\mathrm{R}$ & 0.6 & 0.7 & 0.5 & 0.6 & -0.4 & -0.1 & -0.5 & -0.3 & -0.7 & -0.4 & -0.4 \\
\hline & $\mathrm{P}<$ & 0.0174 & 0.0030 & 0.0311 & 0.0213 & 0.1720 & 0.7420 & 0.0397 & 0.2390 & 0.0038 & 0.1090 & 0.1490 \\
\hline & $\mathrm{n}$ & 16 & 16 & 16 & 16 & 16 & 16 & 16 & 16 & 16 & 16 & 16 \\
\hline \multirow[t]{3}{*}{ ACTA1 } & $\mathrm{R}$ & & 0.6 & 0.9 & 0.9 & -0.5 & -0.4 & -0.6 & -0.3 & -0.6 & -0.4 & -0.4 \\
\hline & $\mathrm{P}<$ & & 0.0104 & 0.0001 & 0.0001 & 0.0700 & 0.1800 & 0.0213 & 0.2180 & 0.0238 & 0.1280 & 0.1260 \\
\hline & $\mathrm{n}$ & & 16 & 16 & 16 & 16 & 16 & 16 & 16 & 16 & 16 & 16 \\
\hline \multirow[t]{3}{*}{ MYH1 } & $\mathrm{R}$ & & & 0.8 & 0.8 & -0.1 & -0.3 & -0.4 & -0.2 & -0.6 & -0.2 & -0.1 \\
\hline & $\mathrm{P}<$ & & & 0.0001 & 0.0002 & 0.6280 & 0.2760 & 0.0874 & 0.4630 & 0.0179 & 0.4030 & 0.5860 \\
\hline & $\mathrm{n}$ & & & 16 & 16 & 16 & 16 & 16 & 16 & 16 & 16 & 16 \\
\hline \multirow[t]{3}{*}{ MYH3 } & $\mathrm{R}$ & & & & 1.0 & -0.4 & -0.6 & -0.7 & -0.4 & -0.7 & -0.4 & -0.4 \\
\hline & $\mathrm{P}<$ & & & & 0.0001 & 0.1310 & 0.0233 & 0.0055 & 0.1410 & 0.0047 & 0.1310 & 0.1480 \\
\hline & $\mathrm{n}$ & & & & 16 & 16 & 16 & 16 & 16 & 16 & 16 & 16 \\
\hline \multirow[t]{3}{*}{ MYH8 } & $\mathrm{R}$ & & & & & -0.5 & -0.6 & -0.7 & -0.4 & -0.8 & -0.5 & -0.5 \\
\hline & $\mathrm{P}<$ & & & & & 0.0412 & 0.0116 & 0.0009 & 0.0965 & 0.0007 & 0.0374 & 0.0474 \\
\hline & $\mathrm{n}$ & & & & & 16 & 16 & 16 & 16 & 16 & 16 & 16 \\
\hline
\end{tabular}

irMYH, fusion index of MYH1-positive myotubes; $n$, number of samples; P, significance; $R$, correlation coefficient. Results in bold indicate a correlation which was not statistically significant. Results from three independent experiments are shown.

NormFinder. Table I shows the order of ranking of the six candidate RGs using NormFinder to calculate their expression stability. Again, both PPIA and B2M were found to be the most stable genes. NormFinder selected PPIA as the most stable gene, with a stability value of $0.267 \pm 0.087$, while B2M had a value of $0.379 \pm 0.087$ (pooled data from myoblasts treated with and without SMFs) (Table I).
BestKeeper (BK). The BestKeeper results of the RG evaluation are shown in Table II. The most stable RGs were identified by the BK software according to their variability, measured by calculation of the lowest coefficient of variance $(\mathrm{CV} \pm \mathrm{SD})$. In this experiment, PPIA, B2M and TBP with a $\mathrm{CV} \pm \mathrm{SD}$ of $1.27 \pm 0.31,1.89 \pm 0.31$ and $1.94 \pm 0.45$, respectively, were found to be the most stable RGs (Table II). RPLPO, 
GAPDH and ACTB exhibited the highest $\mathrm{CV} \pm \mathrm{SDs}$ of $2.00 \pm 0.33,2.06 \pm 0.33$ and $3.26 \pm 0.36$, respectively, indicating that these were the least stable RGs (Table II). However, genes with an SD $>1$ (= starting template variation by the factor 2$)$ should be considered unacceptable. A low SD of the crossing point (CP) values should be expected for suitable RGs. Corresponding to the estimation of the $\mathrm{SD}( \pm \mathrm{CP})$ of the $\mathrm{CV}$ $(\% \mathrm{CP})$, the value was highest for ACTB and GAPDH.

Evaluation of selected candidate RGs and normalization approach. Following identification of the most stable RGs and the normalization factor (NF) from the full panel of RGs, a method was required for their evaluation. Correlations among the myoblast differentiation status (identified by the irMYH1 fusion index) and the expression of ACTA1, MYH1, MYH3 and MYH8 [normalized with NF (PPIA+B2M)] were detected, as shown in Table III. We therefore expected these observations to be mirrored at the mRNA level. More specifically, following treatment with SMFs and DM, a high and significant correlation between the irMYH1 and the gene expression of ACTA1, MYH1, MYH3 and MYH8 was found (Table III), whereas neither the most stable RGs (PPIA and B2M) nor RPLPO, TBP or GAPDH showed a correlation with irMYH1 (Table III, results in bold). A significant positive correlation was found between the genes of interest (GOI), whereas there was no correlation between the RG and the GOI (Table III).

The effect on GOI expression measurements with a good and a poor RG is shown in Fig. 4.

\section{Discussion}

In vitro generation of human functional skeletal muscle tissue with subsequent transfer into a patient is one of the major difficulties encountered in the use of skeletal muscle tissue engineering. The use of satellite stem cells is common in the skeletal muscle tissue engineering approach (9). These cells are easily obtained through muscle biopsies, can be cultivated and expanded in vitro, and mature into multinucleate skeletal muscle tissue. Understanding and thus inducing the differentiation process of mononucleate satellite cells into multinucleate myotubes has promising potential for the regeneration of lost skeletal muscle. Various stimuli which promote the highly complex phenomenon of myogenesis have been identified (neuronal co-cultivation, Matrigel and gene transfer methods), but show great deficits in clinically approved applications (16). Therefore, the identification of strong stimuli, capable of inducing the process of differentiation in human stem cells after cell expansion, is a fundamental issue in skeletal muscle tissue engineering.

Coletti et al reported that stimulation of the rat cell line L6 with SMFs enhanced muscle differentiation in vitro (11). The authors demonstrated that, under the influence of a $\pm 80 \mathrm{mT}$ strong SMF generated by neodymium magnets, the number of mononucleate myoblasts that fused into multinucleate myotubes increased significantly. Additionally, an increased accumulation of actin and myosin and the formation of large myotubes was observed. Therefore, it is of great interest to ascertain whether this effect of SMFs also promotes the maturation of human satellite cells under the same experimental conditions. In order to measure the effects of SMFs on the differentiation of human satellite cell cultures using qRT-PCR, valid RGs must first be identified.

During the complex process of skeletal muscle differentiation, multiple muscle-specific genes are activated at different points, which leads to the fusion of mononucleate myoblasts into multinucleate myotubes (17). During this event, the expression of genes and proteins can be up- or under-regulated, with the changes being drastic or almost undetectable. Stimulation with a strong stimulus such as SMFs, which has been shown to influence the maturation of skeletal muscle cells, additionally affects gene expression. Therefore, the identification of suitable RGs with a constant expression under these conditions for normalization during qRT-PCR experiments is imperative (18).

For this reason, we evaluated six candidate RGs for normalizing gene expression in human satellite cell cultures during stimulation with SMFs. We demonstrated that only PPIA and B2M showed constant expression with and without stimulation by SMFs in human satellite cell cultures. Consequently, only these two genes, determined by three independent software programs [geNorm (13), NormFinder (14) and BestKeeper (15)], fulfill the criterion of expression stability between samples and can be recommended as accurate normalizers for relative gene quantification in cultured satellite cells stimulated by SMFs.

\section{Acknowledgements}

The authors would like to thank Michael Collins for his great help with the manuscript and Petra Prohaska, Ulrike Traut and Rebecca Hollender for their excellent technical assistance.

\section{References}

1. Dheda K, Huggett JF, Bustin SA, Johnson MA, Rook G and Zumla A: Validation of housekeeping genes for normalizing RNA expression in real-time PCR. Biotechniques 37: 112-114, 116, 118-119, 2004

2. Hendriks-Balk MC, Michel MC and Alewijnse AE: Pitfalls in the normalization of real-time polymerase chain reaction data. Basic Res Cardiol 102: 195-197, 2007.

3. Rhinn H, Marchand-Leroux C, Croci N, Plotkine M, Scherman D and Escriou V: Housekeeping while brain's storming validation of normalizing factors for gene expression studies in a murine model of traumatic brain injury. BMC Mol Biol 9: 62, 2008.

4. Dheda K, Huggett JF, Chang JS, Kim LU, Bustin SA, Johnson MA, Rook GA and Zumla A: The implications of using an inappropriate reference gene for real-time reverse transcription PCR data normalization. Anal Biochem 344: 141-143, 2005.

5. Szabo A, Perou CM, Karaca M, Perreard L, Palais R, Quackenbush JF and Bernard PS: Statistical modeling for selecting housekeeper genes. Genome Biol 5: R59, 2004.

6. Huggett J, Dheda K, Bustin S and Zumla A: Real-time RT-PCR normalisation; strategies and considerations. Genes Immun 6: 279-284, 2005.

7. Mahoney DJ, Carey K, Fu MH, Snow R, Cameron-Smith D, Parise G and Tarnopolsky MA: Real-time RT-PCR analysis of housekeeping genes in human skeletal muscle following acute exercise. Physiol Genomics 18: 226-231, 2004.

8. Bustin SA: Absolute quantification of mRNA using real-time reverse transcription polymerase chain reaction assays. J Mol Endocrinol 25: 169-193, 2000.

9. Stern-Straeter J, Bran G, Riedel F, Sauter A, Hormann K and Goessler UR: Characterization of human myoblast cultures for tissue engineering. Int J Mol Med 21: 49-56, 2008.

10. Stern-Straeter J, Riedel F, Bran G, Hormann K and Goessler UR: Advances in skeletal muscle tissue engineering. In Vivo 21: 435-444, 2007. 
11. Coletti D, Teodori L, Albertini MC, Rocchi M, Pristera A, Fini M, Molinaro M and Adamo S: Static magnetic fields enhance skeletal muscle differentiation in vitro by improving myoblast alignment. Cytometry A 71: 846-856, 2007.

12. Machida S, Spangenburg EE and Booth FW: Primary rat muscle progenitor cells have decreased proliferation and myotube formation during passages. Cell Prolif 37: 267-277, 2004.

13. Vandesompele J, De Preter K, Pattyn F, Poppe B, van Roy N, De Paepe A and Speleman F: Accurate normalization of real-time quantitative RT-PCR data by geometric averaging of multiple internal control genes. Genome Biol 3: RESEARCH0034, 2002.

14. Andersen CL, Jensen JL and Orntoft TF: Normalization of real-time quantitative reverse transcription-PCR data: a modelbased variance estimation approach to identify genes suited for normalization, applied to bladder and colon cancer data sets. Cancer Res 64: 5245-5250, 2004.
15. Pfaffl MW, Tichopad A, Prgomet $C$ and Neuvians TP: Determination of stable housekeeping genes, differentially regulated target genes and sample integrity: BestKeeper - Excelbased tool using pair-wise correlations. Biotechnol Lett 26: 509-515, 2004.

16. Stern-Straeter J, Bach AD, Stangenberg L, Foerster VT, Horch RE, Stark GB and Beier JP: Impact of electrical stimulation on three-dimensional myoblast cultures - a real-time RT-PCR study. J Cell Mol Med 9: 883-892, 2005.

17. Brand-Saberi B and Christ B: Genetic and epigenetic control of muscle development in vertebrates. Cell Tissue Res 296: 199-212, 1999.

18. Stern-Straeter J, Bonaterra GA, Hormann K, Kinscherf R and Goessler UR: Identification of valid reference genes during the differentiation of human myoblasts. BMC Mol Biol 10: 66-75, 2009 